\title{
No disk needed around HD 199143 B
}

\author{
G. Chauvin $^{1}$, T. Fusco ${ }^{2}$, A.-M. Lagrange ${ }^{1}$, D. Mouillet $^{3}$, J.-L. Beuzit ${ }^{1}$, M. Thomson ${ }^{1,4}$, J.-C. Augereau $^{5}$, \\ F. Marchis ${ }^{6}$, C. Dumas $^{7}$, and P. Lowrance ${ }^{7}$
}

${ }^{1}$ Laboratoire d'Astrophysique, Observatoire de Grenoble, 414 rue de la piscine, BP 53, 38041 Grenoble Cedex 9, France

2 ONERA, Département d'Optique Théorique et Appliquée, BP 72, 92322 Châtillon Cedex, France

3 Laboratoire d'Astrophysique, Observatoire Midi-Pyrénées, BP 826, 65008 Tarbes, France

${ }^{4}$ Imperial College of Science, Technology and Medecine, Exhibition Road, London SW7 2AZ, England, UK

5 DSM/DAPNIA/Service d'Astrophysique, CEA/Saclay, 91191 Gif-sur-Yvette, France

${ }^{6}$ University of California Berkeley/Center for Adaptive Optics, 601 Campbell Hall, Berkeley, CA 94720, USA

7 Jet Propulsion Laboratory, Mail Stop 183-501, California Institute of Technology, 4800 Oak Grove Drive, Pasadena, CA 91109, USA

Received 26 February 2002 / Accepted 30 July 2002

\begin{abstract}
We present new, high angular resolution images of HD 199143 in the Capricornus association, obtained with the adaptive optics system ADONIS+SHARPII at the ESO $3.6 \mathrm{~m}$ Telescope of La Silla Observatory. HD 199143 and its neighbour star HD 358623 (separation $\sim 5^{\prime}$ away) have previously been imaged with adaptive optics. For each star, a companion has been detected in the $J$ and $K$ bands at respective separations of 1.1" and 2.2" (Jayawardhana \& Brandeker 2001). Our new photometry of HD 199143 B suggests that it is a M2 star and that the presence of circumstellar dust proposed by van den Ancker et al. (2000) is no longer necessary. We show that the $12 \mu \mathrm{m}$ flux detected by IRAS previously interpreted as an IR excess, can be explained by the presence of the late-type companion.
\end{abstract}

Key words. stars: imaging - binaries: general - stars: low-mass, brown dwarfs - stars: pre-main sequence

\section{Introduction}

During the last few years, the nearby young stellar associations have become important targets for studying disk evolution and planet formation (Jayawardhana \& Greene 2001). Indeed, their proximity (typically closer than $100 \mathrm{pc}$ ) makes them ideal to observe the close circumstellar environment. Their youth (around $10 \mathrm{Myr}$ ) implies that planets may have already formed and that circumstellar disks may be optically thin and show evidence of spatial structure such as rings and gaps. The recent observations of the TW Hydrae Association (Kastner et al. 1997; Webb et al. 1999) or the Tucana Association (Zuckerman et al. 2000; Zuckerman et al. 2001a) have revealed the existence of low-mass companions or circumstellar disks in these young and nearby open clusters. These detections are crucial to constrain the current models of proto-planetary systems formation and evolution. Searching more deeply into the close circumstellar environments of known associations and prospecting for new open clusters is clearly needed. The two physically bound stars HD 199143 and HD 358623 have recently been presented as a new possible young association (the Capricornus Association), located at $\sim 48 \mathrm{pc}$ and aged at $\sim 10 \mathrm{Myr}$ (van den Ancker et al. 2000). They also have been

Send offprint requests to: G. Chauvin,

e-mail: gchauvin@obs.ujf-grenoble.fr associated recently to the Beta Pictoris Group by Zuckerman et al. (2001b). Spectroscopic observations have revealed the presence of ultraviolet and IR excess for HD 199143 which were attributed to the presence of an accretion disk around an hypothetical T Tauri-like companion.

To test these interpretations, high resolution imaging was needed. The first adaptive optics (AO) observations of the Capricornus association were carried out on 2001, May 31th and June 1st. Two companions were resolved around both HD 199143 and HD 358623 (Jayawardhana \& Brandeker 2001). In the case of the binary system HD 199143 , the $(J-K=$ 1.37) color observed for HD $199143 \mathrm{~B}$ was attributed to the likely presence of a circumstellar disk. We present in this paper new results in $J, H$ and $S K$ (short $K$ ) bands for this source, which lead to a different interpretation of the spectral type of HD 199143 B and the possible existence of a circumstellar disk around this companion.

\section{Observation and data reduction}

The observations were conducted at the La Silla Observatory on the $3.6 \mathrm{~m}$ telescope on 2001, June 2nd and 3thd and October 28th and 29th. The AO system ADONIS combined with the SHARPII camera was used to observe HD 199143 in the near infrared broad bands $J, H$ and $S K$. A plate scale of $0.05^{\prime \prime} /$ pixel (field of view of $12.8^{\prime \prime} \times 12.8^{\prime \prime}$ ) was chosen. In June, about 
100 frames were taken with individual integration times of $0.4 \mathrm{~s}$ in $J, 0.3 \mathrm{~s}$ in $H$ and $0.3 \mathrm{~s}$ in $S K$ with a seeing ranging from $0.85^{\prime \prime}$ to $1.5^{\prime \prime}$ in $V$ band. In October, we obtained 100 frames of $0.2 \mathrm{~s}$ in $H$ and $S K$ and 100 frames of $0.3 \mathrm{~s}$ in $J$ under good seeing conditions (stable at $0.9^{\prime \prime}$ ).

The reference star HD 198420 was observed to estimate the PSF in each band during both periods. We also observed the stars S055D, S294D and S209D, taken from the HST photometric standards catalog, to estimate the PSF and obtain the near IR photometry of HD 199143 A and its companion HD 199143 B.

The data were corrected from detector and sky background effects (bias, flat-fielding and badpixels correction). Then, the myopic deconvolution algorithm MISTRAL (Fusco et al. 1999; Conan et al. 2000) was used to obtain the flux ratio and the separation of the system HD 199143. MISTRAL allows the restoration of both the object and the Point Spread Function (PSF). This technique is called "myopic" deconvolution because it accounts for the uncertainties and variability of the PSF through the use of several images of a reference star. The a priori knowledge of the object structure (multiple star) is also used. Figure 1 presents the result of the deconvolution on $J$ band images.

\section{Results and comparison to previous data}

Based on the observations obtained in October 2001 (under the best photometric and seeing conditions), we derived the photometry of HD 199143 A and its companion HD 199143 B in $J$, $H$ and $K$ bands. To obtain the photometry in $K$ band, we had to consider a correction factor to convert the flux from $S K$ to $K$ for both stars. We took into account the spectral transmissions of both filters and the difference of spectral type between the science object (F8V star HD 199143 A and M2 star HD 199143 B) and an A0V star. In both cases, this factor was smaller than 0.004 mag and therefore neglected. The separation between the two components was estimated by MISTRAL at $1.074 \pm 0.004^{\prime \prime}$ (consistent in $J, H$ and $K$ for observations of June and October 2001). The new photometric results are reported in Table 1. The contrasts between HD 199143 A and B during the three AO observations are also compared in Table 2. Jayawardhana \& Brandeker (2001) used the myopic deconvolution algorithm IDAC for their data set. For HD 199143 A, our results are con-
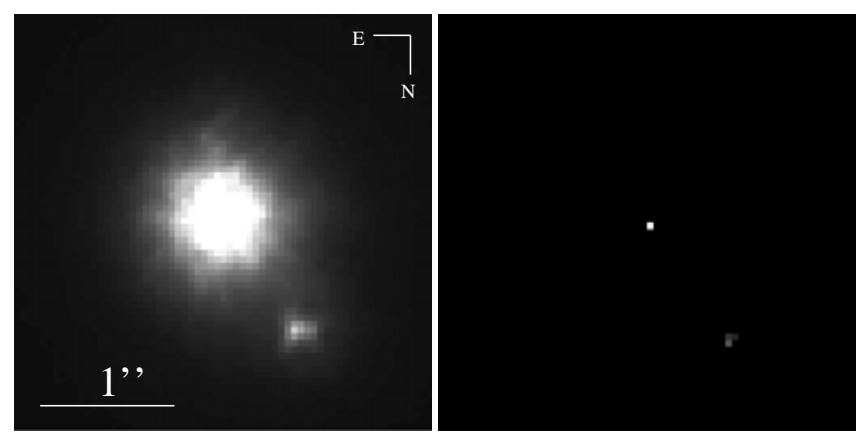

Fig. 1. Left: ADONIS image in $J$ band of HD 199143 A and B (the pixel scale is 50 mas). Right: resulting image obtained with the MISTRAL myopic deconvolution algorithm.
Table 1. Photometry of HD 199143 A and B (October 2001).

\begin{tabular}{llll}
\hline \hline Sources & $\begin{array}{l}J \\
(\mathrm{mag})\end{array}$ & $\begin{array}{l}H \\
(\mathrm{mag})\end{array}$ & $\begin{array}{l}K \\
(\mathrm{mag})\end{array}$ \\
\hline HD 199143 A + B & 6.19 & 5.90 & 5.82 \\
HD 199143 A & 6.27 & 6.02 & 5.95 \\
HD 199143 B & 8.95 & 8.35 & 8.14 \\
\hline
\end{tabular}

Table 2. Comparaison of actual contrast observations between HD 199143 A and B.

\begin{tabular}{lllll}
\hline \hline Epoch & $\Delta J$ & $\Delta H$ & $\Delta K$ & Dec Algo. \\
\hline $01 / 06^{a}$ & $3.25 \pm 0.04$ & - & $2.21 \pm 0.03$ & IDAC \\
$02 / 06^{b}$ & $2.67 \pm 0.05$ & - & $2.16 \pm 0.02$ & MISTRAL \\
$29 / 10^{c}$ & $2.68 \pm 0.03$ & $2.33 \pm 0.01$ & $2.19 \pm 0.02$ & MISTRAL \\
\hline
\end{tabular}

${ }^{a}$ (Jayawardhana \& Brandeker 2001).

${ }^{b}$ (this paper).

(this paper).

sistent with the values obtained by Jayawardhana \& Brandeker (2001) respectively $J=6.23 \pm 0.04$ and $K=5.90 \pm 0.03$ if we consider our photometric uncertainties of $0.04 \mathrm{mag}$. These results are also consistent with the photometric values derived for unresolved observations of HD 199143 presented by Oudmaijer et al. (2001).

But, interestingly, the contrasts we measured between HD 199143 A and B at $J$ band are significantly different from those measured by Jayawardhana \& Brandeker (2001) as we will discuss in the next section.

\section{Discussion of contrast estimation}

In the case of HD 199143 B, our photometry presented in Table 1 is different from the one presented by Jayawardhana \& Brandeker (2001) as we found a significant difference of contrast in $J$ band. This difference is supported by the results obtained in June and October 2001. Instrumental effects can be dismissed because the observations were taken with the same instrumental configuration (ADONIS+SHARPII) on the ESO $3.6 \mathrm{~m}$ telescope. Uncertainties in our photometric measurements cannot be the source of this 0.57 mag difference since our $K$-band measurements are in perfect agreement between the two observation dates (02/06 and 29/10). Moreover, the $J$ and $K$ band contrast measurements that we obtained between HD 358623 A and HD 358623 B are consistent with the results of Jayawardhana \& Brandeker (2001) as presented in Table 3. In this case, the separation between the two components is estimated at $\sim 2.2^{\prime \prime}$ and is more favorable for the deconvolution. We propose to investigate the two causes which may be responsible for this discrepancy: the variability of the stars and the two myopic deconvolution algorithms used IDAC and MISTRAL. 
Table 3. Comparaison of actual contrast observations between HD $358623 \mathrm{~A}$ and B.

\begin{tabular}{llll}
\hline \hline Epoch & $\Delta J$ & $\Delta K$ & Dec Algo. \\
\hline $01 / 06^{a}$ & $1.81 \pm 0.08$ & $1.67 \pm 0.06$ & IDAC \\
$02 / 06^{b}$ & $1.75 \pm 0.06$ & $1.67 \pm 0.05$ & MISTRAL \\
\hline
\end{tabular}

${ }^{a}$ (Jayawardhana \& Brandeker 2001).

${ }^{b}$ (this paper).

\subsection{Variability or flaring}

A first explanation might be the photometric variability of one or both stars in this sytem; this phenomenon is relatively frequent for young $\mathrm{T}$ Tauri stars. However, Carpenter et al. (2001) have observed near-infrared photometric variability of Classical and Weak T Tauri stars toward the Orion A molecular cloud. The mean peak to peak amplitudes they detected were $\sim 0.2 \mathrm{mag}$, less than the present difference of $0.57 \mathrm{mag}$. In addition, even if the case of a flaring star is considered, a variability observed in $J$ band would also be expected in $K$ band which is not the case. Consequently, it is unlikely that this difference originates from stellar processes.

\subsection{Deconvolution algorithms}

The discrepancy of the $J$ band photometry is present between two distinct data sets deconvolved with two different deconvolution algorithms MISTRAL (that we used) and IDAC (used by Jayawardhana \& Brandeker 2001). We first investigated the difference in the image quality of both observations as shown in Fig. 2. Jayawardhana \& Brandeker (2001) kindly shared their data to conduct this analysis. The AO correction in their observations is low compared to our data (due probably to worse weather conditions). Our observations are then more favorable to perform myopic deconvolution and deduce the photometry of HD 199143 B (see Table 1). If we perform deconvolution with IDAC on our observation, the results are consistent with MISTRAL deconvolution results (see Table 4).

In the unfavorable case of a low AO correction, we can still perform deconvolution but the regularisation on the PSF and the object needs to be done cautiously. The results obtained with MISTRAL and IDAC on Jayawardhana \& Brandeker (2001) observations are also presented in Table 4 and show that this regularisation seems to be responsible for the discrepancy in $J$ band.

\section{Astrophysical results}

\section{1. $H D 199143$ A}

With our new photometric measurements, we have investigated the evolutionary status of HD 199143 A. Figure 3 shows the colors of HD 199143 A overplotted on the isochrones provided by Baraffe et al. (2002). We thus derive the two possible stellar parameters of this star. The results are presented in Table 5.

These parameters are consistent with an expected F8V spectral type (Schmidt-Kaler et al. 1982; van den Ancker et al. 2000). The age found for HD $199143 \mathrm{~A}$ is older than $18 \pm 2 \mathrm{Myr}$

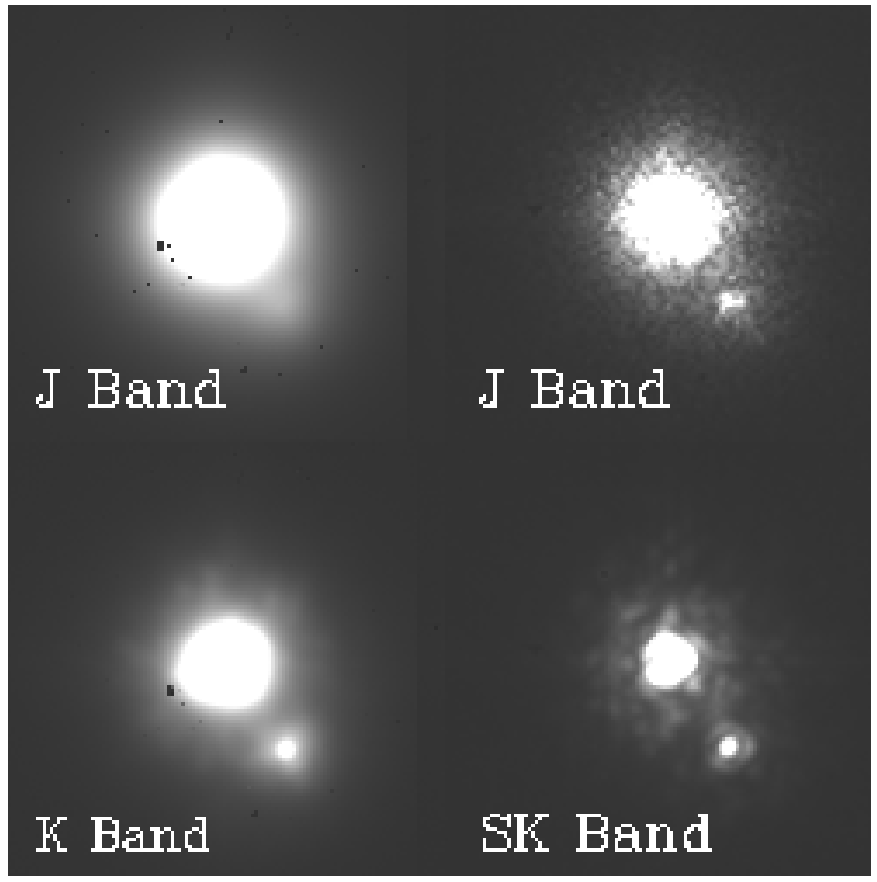

Fig. 2. Left: ADONIS images in $J$ and $K$ band of HD 199143 AB obtained by Jayawardhana \& Brandeker (2001). Right: ADONIS images we have obtained in $J$ and $S K$. The images are scaled to the same pixel scale (35 mas) and the same integration time (1 s).

Table 4. MISTRAL and IDAC results compared for the observations obtained by Jayawardhana \& Brandeker (2001) in (01/06) and our observations in (29/10) in the case of HD 199143.

\begin{tabular}{llll}
\hline \hline Epoch & $\Delta J$ & $\Delta K$ & Dec Algo. \\
\hline $01 / 06^{a}$ & $3.25 \pm 0.04$ & $2.21 \pm 0.03$ & IDAC \\
$01 / 06^{a}$ & $2.73 \pm 0.1$ & $2.14 \pm 0.1$ & MISTRAL \\
\hline $29 / 10^{c}$ & $2.72 \pm 0.05$ & $2.25 \pm 0.05$ & IDAC \\
$29 / 10^{c}$ & $2.68 \pm 0.03$ & $2.19 \pm 0.02$ & MISTRAL \\
\hline
\end{tabular}

a (Jayawardhana \& Brandeker 2001).

${ }^{c}$ (this paper).

when compared to the estimation reported by Jayawardhana \& Brandeker (2001), but in reasonable agreement if we consider the uncertainty of the color measurements and of the stellar model we used. If we consider the evolutionary tracks $M_{K}$ versus $J-K$ and $M_{H}$ versus $J-H$ which most constrain the age of HD 199143 A, we see that this object is likely to be aged of 25 Myr. However, an age of 31 Myr is still possible according to the uncertainties. We then considered both ages to derive the stellar parameters presented Table 5 .

\section{2. $H D 199143 B$}

From the color analysis proposed by Jayawardhana \& Brandeker (2001), HD 199143 B appeared to be extremely red. They attributed the strong $J-K$ color of 1.37 mag to an IR excess caused by a circum-secondary disk around HD 199143 B, as first suggested by van den Ancker et al. (2000) based on $12 \mu \mathrm{m}$ IRAS excess. Our more recent observations (Table 1) 

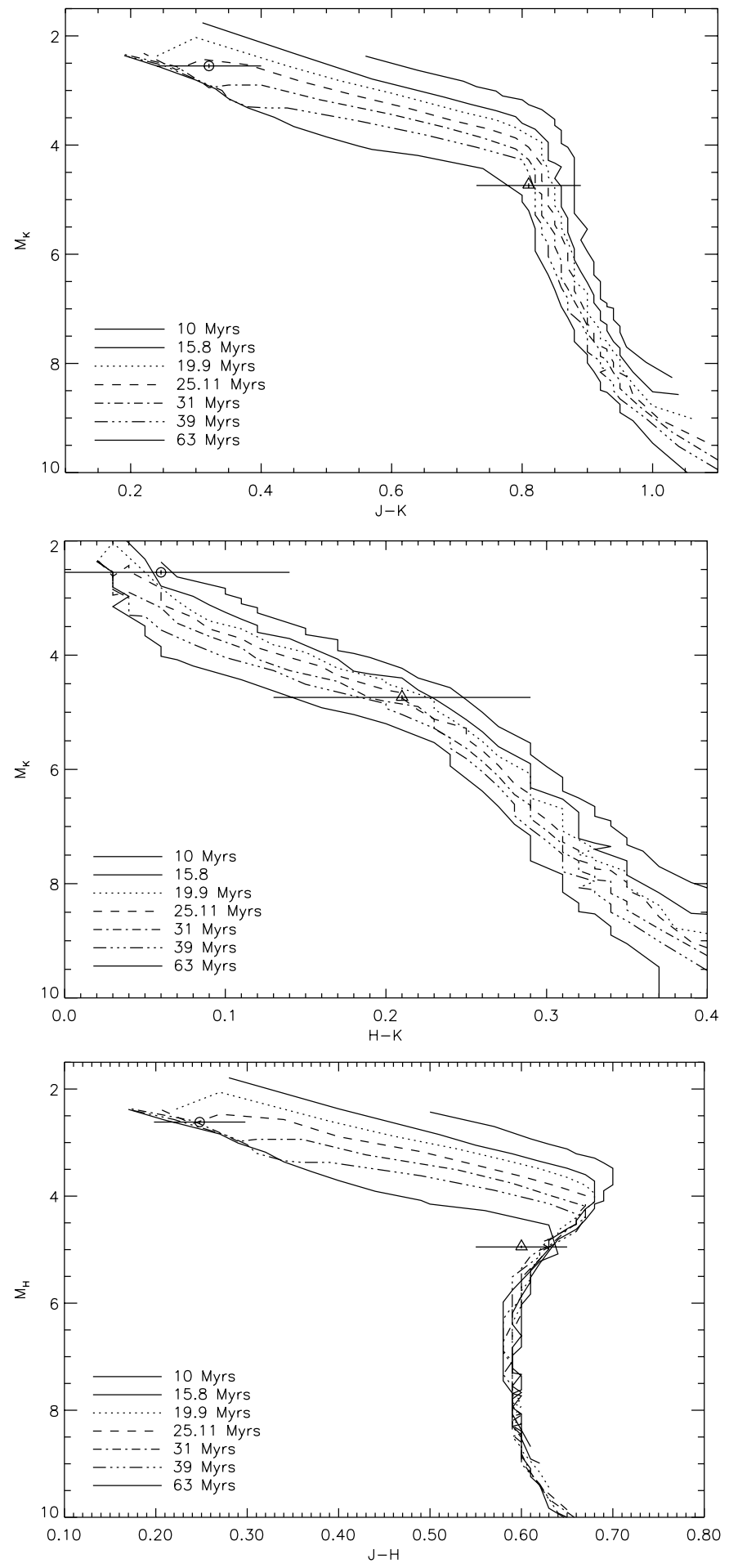

Fig. 3. Comparison of evolutionnary tracks given by Baraffe et al. (2002) representing absolute Magnitudes versus colors with the measurements of HD 199143 A (circle) and HD 199143 B (triangle). Uncertainties on the colors measurements are also overplotted. We supposed that the system was located at $48 \mathrm{pc}$ with no extinction (van den Ancker et al. 2000; Jayawardhana \& Brandeker 2001).

fail to support the need for this interpretation as HD 199143 B is significantly less red $(J-K=0.81)$. As we suppose that the components HD $199143 \mathrm{~A}$ and B are coeval, the HD 199143 B age is 25 or 31 Myr. Using the evolutionary
Table 5. Stellar parameters derived from the isochrones provided by Baraffe et al. (2002) for an age of $25 \mathrm{Myr}$ and $31 \mathrm{Myr}$.

\begin{tabular}{lllll}
\hline \hline \multicolumn{5}{c}{ Age of 25 Myr } \\
\hline Source & $M$ & $T_{\text {eff }}$ & $\log (g)$ & $L$ \\
& $\left(M_{\odot}\right)$ & $(\mathrm{K})$ & $\left(L_{\odot}\right)$ \\
\hline HD 199143 A $[1.2,1.3]$ & {$[5770,5990]$} & {$[4.17,4.29]$} & {$[2.18,2.23]$} \\
HD 199143 B $[0.57,0.6]$ & {$[3622,3651]$} & {$[4.46,4.45]$} & {$[0.08,0.09]$} \\
\hline \multicolumn{5}{c}{ Age of 31 Myr } \\
\hline \multicolumn{5}{c}{$T_{\text {eff }} \log (g)$} \\
\hline Source & $M$ & $L$ \\
& $\left(M_{\odot}\right)$ & $(\mathrm{K})$ & $\left(L_{\odot}\right)$ \\
\hline HD 199143 A 1.3 & 6046 & 4.28 & 2.24 \\
HD 199143 B $[0.6,0.62]$ & {$[3661,3683]$} & {$[4.51,4.50]$} & {$[0.08,0.09]$} \\
\hline \multicolumn{5}{c}{}
\end{tabular}

model of Baraffe et al. (2002), we derived the stellar parameters for HD 199143 B as for HD 199143 A. The results are presented in Table 5. These values are consistent with the IR colors of a M2 star according to the color table given by Kenyon et al. (1995). The presence of circumstellar matter is no longer needed to explain the color measurements of this object.

\section{Origin of the $12 \mu \mathrm{m}$ excess}

If a circumsecondary disk is no longer needed to explain the near-IR observations of HD $199143 \mathrm{~B}$, one question is still unsolved: what is the source of the $12 \mu \mathrm{m}$ excess detected by van den Ancker et al. (2000) from IRAS data? In their spectral analysis, van den Ancker et al. (2000) fitted the optical measurements of the HD 199143 A+B by a single Kurucz spectra $\left(T_{\text {eff }}=6200 \mathrm{~K}\right.$ and $\left.\log g=4.3\right)$. Extrapolating this spectrum to IR wavelengths, they deduced the existence of an IR excess only at $12 \mu \mathrm{m}$ of $0.24 \pm 0.04 \mathrm{Jy}$. At 25,60 and $100 \mu \mathrm{m}$, respective upper limits of $0.12,0.12$ and $0.3 \mathrm{Jy}$ were obtained from IRAS data.

If we now consider that HD 199143 is a binary system with a F8 primary star and a M2 companion, the spectral contribution of the two stars can be adjusted using optical and near-IR measurements. Figure 4 shows Kurucz (1991) spectra of a $5900 \mathrm{~K}$ star for HD $199143 \mathrm{~A}$ and of a $3600 \mathrm{~K}$ star for HD 199143 B. Their sum and observed measurements (circles) are also plotted. If we compare the sum of the two spectral contributions of HD 199143 A and HD 199143 B to the IRAS measurement at $12 \mu \mathrm{m}$, the IR excess is no longer obvious. Indeed, considering the uncertainty on the IRAS flux, the spectral sum of the two components is consistent with the value of $0.24 \pm 0.04 \mathrm{Jy}$. Therefore, the IRAS $12 \mu \mathrm{m}$ observation can be explained by a late spectral type for HD 199143 B. Hence, the presence of dust in this system is no longer necessary to explain near-IR and mid-IR measurements. 


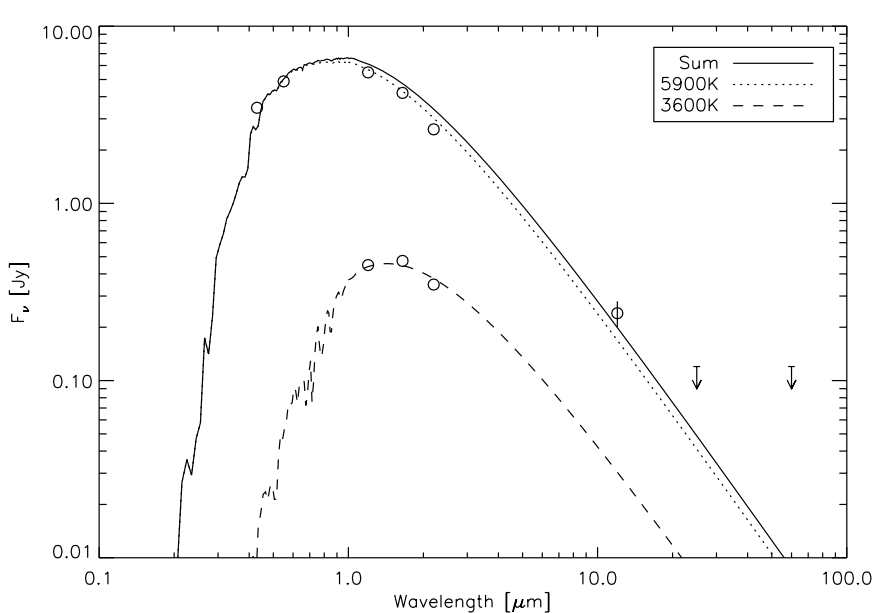

Fig. 4. Observed fluxes of HD 199143 A and B in optical, near-IR and mid-IR (circles) compared to Kurucz spectra of a $5900 \mathrm{~K}$ star, of a $3600 \mathrm{~K}$ star and of their sum.

\section{Conclusions}

We have presented new adaptive optics images of the binary system HD 199143 AB from the Capricornus Association. They confirm the two components of this source, as resolved by Jayawardhana \& Brandeker (2001), but return a different contrast in $J$ band. We discussed the possible causes of this difference (photometric variability, AO correction, deconvolution algorithms). By comparing the results obtained with IDAC and MISTRAL deconvolution techniques, we found that the data are consistent with observations obtained under good AO correction. For less favorable conditions, the regularisation of the PSF and/or the object in the deconvolution process is critical and may be responsible for the discrepancy between the two algorithms on the observations of Jayawardhana \& Brandeker (2001).

Our new results confirm the F8 spectral type of HD 199143 A but derive different characteristics for its companion HD 199143 B. From our observations, we have deduced that HD 199143 B is more likely to be a M2 star. From the evolutionnary model of Baraffe et al. (2002), we have derived the stellar parameters for the two stars and found an estimation for the age of the system between $25 \mathrm{Myr}$ and $31 \mathrm{Myr}$, slightly higher than Jayawardhana \& Brandeker (2001). We have also found that the IR excess at $12 \mu \mathrm{m}$ is no longer needed because the spectral sum of the components HD 199143 A and HD $199143 \mathrm{~B}$ was consistent with the IRAS flux of $0.24 \pm 0.04 \mathrm{Jy}$. Consequently, we conclude that the existence of the late-type companion HD 199143 B can explain the present near-IR and mid-IR observations.

Acknowledgements. We would like to thank Ray Jayawardhana and Alexis Brandeker who kindly shared their observations. This allowed us to perform tests on both myopic deconvolution algorithms IDAC and MISTRAL and to conduct our discussion on the contrast estimation. We would like to thank also the CNES for the financial support given to Jean-Charles Augereau and finally the staff of the ESO $3.6 \mathrm{~m}$ telescope and particulary Kate Brooks for her outstanding support.

\section{References}

Baraffe, I., Chabrier, G., Allard, F., \& Hauschildt, P. G. 2002, A\&A, 382,563

Carpenter, J. M., Hillenbrand, L. A., \& Skrutskie, M. F. 2001, AJ, 121, 3160

Conan, J.-M., Fusco, T., Mugnier, L., et al. 2000, SPIE, 400, 913

Fusco, T., Véran, J.-P., Conan, J.-M., \& Mugnier, L. 1999, A\&AS, 134,193

Fusco, T. 2000, Correction partielle et anisoplanétisme en Optique Adaptative: traitement a posteriori et Optique Adaptative Multiconjuguée, Thesis

Jayawardhana, R., \& Brandeker, A. 2001, ApJ, 561, L111

Jayawardhana, R., \& Greene, T. 2001, Young Stars Near Earth: Progress and Prospects

Kastner, J. H., Zuckerman, B., Weintraub, D. A., \& Forveille, T. 1997, Science, 277, 67

Kenyon, S. J., \& Hartmann, L. 1995, ApJS, 101, 117

Kurucz, R. L. 1991, in Stellar Atmospheres-Beyond classical Models, ed. A. G. Davis Philip, A. R. Upgren, \& K. A. Janes (L. Davis press, Schenectady, New-York), 441

Oudmaijer, R. D., Palacios, J., Eiroa, C., et al. 2001, A\&A, 379, 564

Schmidt-Kaler, Th. 1982, Landolt-Bornstein Catalogue, VI/2b

Van den Ancker, M. E., Pérez, M. R., \& de Winter, D. 2000, A\&A, 363, L25

Webb, R. A., Zuckerman, B., Platais, I., et al. 1999, ApJ, 512, L63

Zuckerman, B., \& Webb, R. A. 2000, ApJ, 535, 959

Zuckerman, B., Song, I., \& Webb, R. A. 2001a, ApJ, 559, 388

Zuckerman, B., Song, I., Bessel, M. S., \& Webb, R. A. 2001b, ApJ, 562, L87 